\title{
Ajuste cinético de reacciones en serie en la hidrólisis ácida de celulosa y hemicelulosa de cascarilla de arroz
}

\author{
Jorge Sigüencia ${ }^{1}$, Jaime Soler ${ }^{2}$, Miguel Menéndez ${ }^{2}$ \\ ${ }^{1}$ Universidad de Cuenca, 12 de abril y Agustín Cueva, Cuenca, Ecuador \\ ${ }^{2}$ Grupo de Catálisis, Separaciones Moleculares e Ingeniería de Reactores (CREG) \\ ${ }^{2}$ Instituto de Investigación en Ingeniería de Aragón (I3A) \\ ${ }^{2}$ Universidad de Zaragoza, Mariano Esquillor s/n, 50018, Zaragoza, Spain. \\ Tel. +34-876555481, e-mail: jorge.siguencia@ucuenca.edu.ec
}

\section{Resumen}

Se han determinado las constantes cinéticas para el proceso de hidrólisis de cascarilla de arroz con experimentos a diferentes condiciones de operación.

\section{Introducción}

En el Ecuador la producción de arroz en cáscara corresponde aproximadamente a 1,6 millones de toneladas anuales, donde los residuos de cascarilla representan el 22\% del total producido [1]. La hidrólisis ácida de biomasa permite obtener azúcares fermentables utilizables en la generación de biocombustibles. Esto podría suponer una fuente de energía renovable a partir de un residuo agrícola.

Generalmente todos los estudios referidos a hidrólisis de residuos lignocelulósicos abarcan un modelo cinético. La cinética de la hidrólisis ácida es relativamente compleja ya que sucede en varias fases y con gran influencia de las condiciones de operación, temperatura y la concentración del ácido utilizado, por lo general diluido. Para facilitar su estudio se han formulado modelos cinéticos simplificados en términos de reacciones irreversibles pseudo-homogéneas de primer orden que buscan explicar y predecir los cambios estructurales que se dan en los compuestos involucrados en las mismas. El principal referente de estos modelos es el propuesto por Saeman [2] y la principal limitación de los mismos es disponer de los valores de los parámetros cinéticos para un caso de aplicación específico.

El objetivo de este estudio es realizar un ajuste cinético aplicando un modelo de primer orden en serie para calcular k1 y k2 a partir de datos experimentales obtenidos de la manipulación de la temperatura y tiempo de reacción, manteniendo constantes variables como: concentración de ácido sulfúrico y cantidad de biomasa alimentada al reactor.

\section{Metodología}

Se utilizó como biomasa la cascarilla de arroz SFL09 e INIAP 14, con 56\% de celulosa + hemicelulosa en peso.

Para la experimentación se utilizó un reactor tipo batch del $600 \mathrm{ml}$ de capacidad, en el cual se colocó la mezcla al $1 \%$ de cascarilla de arroz y $1 \%$ de $\mathrm{H}_{2} \mathrm{SO}_{4}$. Se varió la temperatura entre $100^{\circ} \mathrm{C}, 120^{\circ} \mathrm{C}$ y $150^{\circ} \mathrm{C}$, así como el tiempo de reacción de 6 a 90 minutos. Se realizaron en total 30 experimentos. Se propone el modelo de reacción en serie [3]:

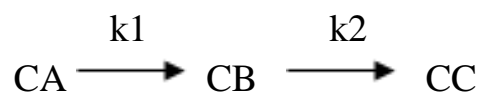

donde, CA, CB y CC representan las concentarciones de (celulosa + hemicelulosa), oligosacáridos y azúcares reductores respectivamente, en $\mathrm{g} / 100 \mathrm{~g}$ de celulosa + $\mathrm{h}$. celulosa soluble, $\mathrm{k}_{1} \mathrm{y} \mathrm{k}_{2}$ representan las constantes cinéticas en $\mathrm{min}^{-1}$, sabiendo que la concentración inicial de A es CAo. Las ecuaciones 1,2 y 3 se utilizaron para el ajuste de las concentraciones

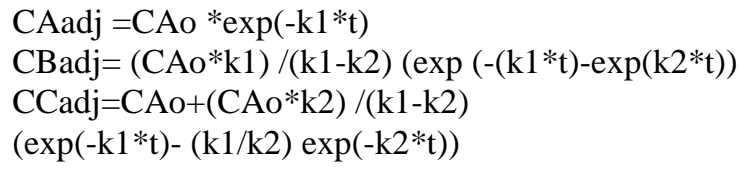

La conversión de (celulosa + hemicelulosa) se determinó mediante el cálculo de $f$ [4] que es la relación entre el carbono orgánico solubilizado a un tiempo dado y el total en la suma de celulosa y hemicelulosa inicial (Ecuación 4). Se considera que $f$ aumenta asintóticamente hasta un valor límite $(f l)$.

$$
\begin{aligned}
& f=\frac{\operatorname{COT}_{\text {salida }}}{\operatorname{COT}_{\text {entrada }}} \\
& f_{a d j}=f l\left(1-e x p^{-k 1 t}\right)
\end{aligned}
$$


La ecuación 5 representa el ajuste de f en función de la máxima conversión posible $f l$. El valor de $f l, \mathrm{k}_{1} \mathrm{y}$ $\mathrm{k}_{2}$ se determinaron utilizando Solver de Excel.

\section{Resultados}

Las constantes de velocidad $\mathrm{k}_{1}, \mathrm{k}_{2}$ incrementan al aumentar la temperatura (Tabla 1). De manera similar lo hace la conversión, llegándose a obtener un nivel máximo de 0,52 para una temperatura de $150^{\circ} \mathrm{C}$ (Figura 3).

Tabla 1: Valores de k1 y k2 obtenidos

\begin{tabular}{|l|l|c|}
\hline $\mathbf{T}\left(\mathbf{~}^{\mathbf{0}} \mathbf{C}\right)$ & $\mathbf{k}_{\mathbf{1}}\left(\mathbf{m i n}^{-1}\right)$ & $\mathbf{k}_{\mathbf{2}}\left(\mathbf{m i n}^{-1}\right)$ \\
\hline 100 & 0,0864 & 0,0151 \\
\hline 120 & 0,206 & 0,018 \\
\hline 150 & 0,235 & 0,025 \\
\hline
\end{tabular}

En cuanto a las concentraciones de CA, CB y CC, se observa que la producción de oligosacáridos al inicio de la reacción es alta, sin embargo, desde el minuto 20 en adelante empieza a declinar por el aumento de la producción de azúcares. (Figura 4).

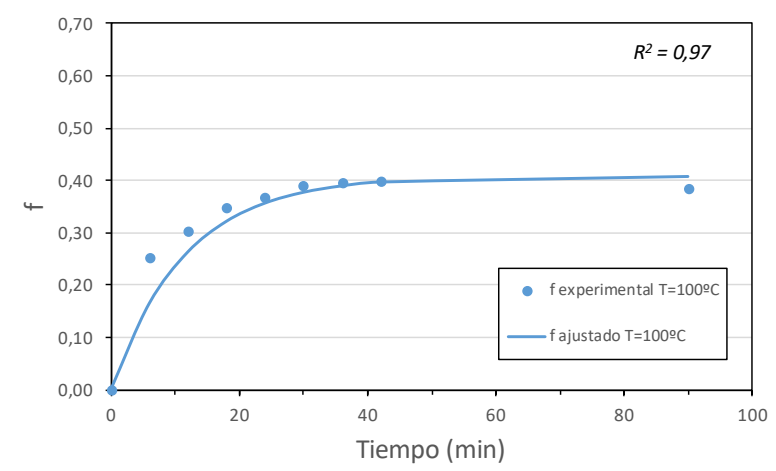

Figura 1: Conversión de celulosa + hemicelulosa a $100^{\circ} \mathrm{C}$

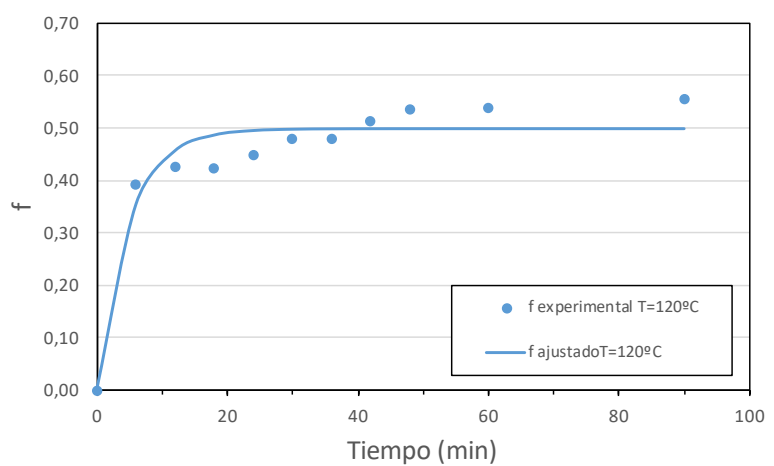

Figura 2: Conversión de celulosa + hemicelulosa a $120^{\circ} \mathrm{C}$

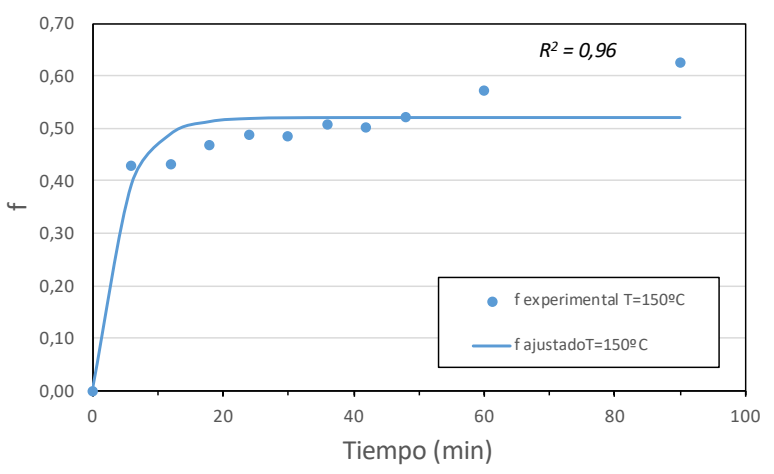

Figura3: Conversión de celulosa + hemicelulosa a $150^{\circ} \mathrm{C}$

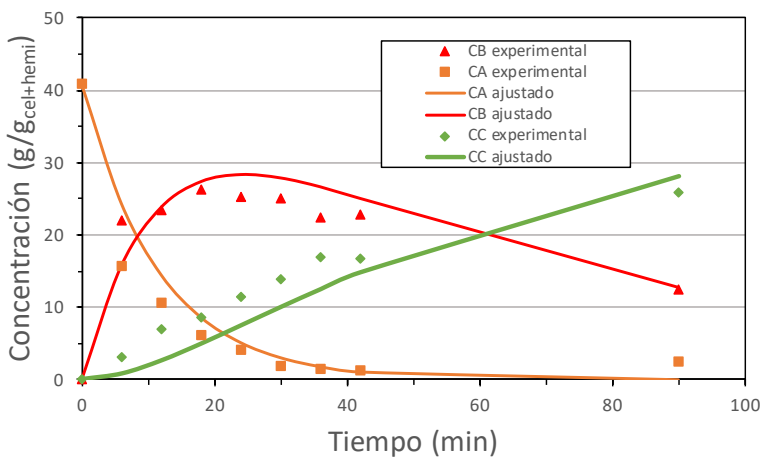

Figura 4: Generación de CA, CB y CC a $100^{\circ} \mathrm{C}, 1 \%$ Biomasa, $1 \%$ ácido

\section{Conclusiones}

La temperatura afecta directamente al aumento de las constantes cinéticas, así como a la generación de azúcares reductores. El modelo cinético de dos reacciones en serie ajusta de buena manera los datos experimentales $\left(R^{2}=0,96\right.$ y 0,97$)$.

\section{REFERENCIAS}

[1] ECHEVERRÍA, M.A. and LÓPEZ, O. Caracterización energética de la cascarilla de arroz para su aplicación en la generación de energía termoeléctrica, Escuela Politécnica Nacional de Ecuador, 2010. Proyecto de Fín de Título.

[2] SAEMAN, J. Kinetics of wood saccharification Hydrolysis of cellulose and decomposition of sugars in dilute acid at high temperature. Industrial Engineering Chem. 1945, 37, 43-52. doi: 10.1021 / ie50421a009.

[3] GARROTE, G., DOMINGUEZ, H. and PARAJO J.C, Kinetic modelling of corn cob autohydrolysis. Process Biochem. 2001, 36, 571-578. doi:10.1016/S00329592(00)00253-3

[4] BRUNER, G. Near critical and supercritical water. Part I Hydrolytic and hydrotermal process, The Journal Supercritical Fluids. 2009, 47, 373-381. doi:10.1016/j.supflu.2008.09.002 